\title{
International genomic definition of pneumococcal lineages, to contextualise disease, antibiotic resistance and vaccine impact
}

\author{
Rebecca A. Gladstone ${ }^{\mathrm{a}, *, 1}$, Stephanie W. Lo ${ }^{\mathrm{a}, 1}$, John A. Lees ${ }^{\mathrm{b}}$, Nicholas J. Croucher ${ }^{\mathrm{c}}$, Andries J. van Tonder ${ }^{\mathrm{a}}$, \\ Jukka Corander ${ }^{\mathrm{a}, \mathrm{d}}$, Andrew J. Page ${ }^{\mathrm{a}}$, Pekka Marttinen ${ }^{\mathrm{e}}$, Leon J. Bentley ${ }^{\mathrm{a}}$, Theresa J. Ochoa ${ }^{\mathrm{f}}$, Pak Leung Ho ${ }^{\mathrm{g}}$, \\ Mignon du Plessis ${ }^{\mathrm{h}}$, Jennifer E. Cornick ${ }^{\mathrm{i}}$, Brenda Kwambana-Adams ${ }^{\mathrm{j}, \mathrm{k}}$, Rachel Benisty ${ }^{1}$, Susan A. Nzenze ${ }^{\mathrm{m}, \mathrm{n}}$, \\ Shabir A. Madhi ${ }^{\mathrm{m}, \mathrm{n}}$, Paulina A. Hawkins ${ }^{\circ}$, Dean B. Everett ${ }^{\mathrm{p}}$, Martin Antonio ${ }^{\mathrm{k}, \mathrm{q}}$, Ron Dagan ${ }^{1}$, Keith P. Klugman ${ }^{\circ}$, \\ Anne von Gottberg ${ }^{\mathrm{h}}$, Lesley McGee ${ }^{\mathrm{r}}$, Robert F. Breiman ${ }^{\mathrm{o}, \mathrm{s}}$, Stephen D. Bentley ${ }^{\mathrm{a}}$, \\ The Global Pneumococcal Sequencing Consortium ${ }^{2}$
}

a Parasites and microbes, Wellcome Sanger Institute, Hinxton, UK

${ }^{\mathrm{b}}$ New York University School of Medicine, New York, NY, USA

${ }^{\mathrm{c}}$ Faculty of Medicine, School of Public Health, Imperial College London, UK

d Department of Biostatistics, University of Oslo, 0317 Oslo, Norway

e Department of Computer Science, Helsinki Institute for Information Technology HIIT, Espoo, Finland

${ }^{\mathrm{f}}$ Instituto de Medicina Tropical, Universidad Peruana Cayetano Heredia, Lima, Peru

g Department of Microbiology, Carol Yu Centre for Infection, Queen Mary Hospital, The University of Hong Kong, Hong Kong, China

h Centre for Respiratory Diseases and Meningitis, National Institute for Communicable Diseases, Johannesburg, South Africa

${ }^{\text {i } M a l a w i-L i v e r p o o l-W e l l c o m e-T r u s t ~ C l i n i c a l ~ R e s e a r c h ~ P r o g r a m m e, ~ B l a n t y r e, ~ M a l a w i ~}$

${ }^{j}$ NIHR Global Health Research Unit on Mucosal Pathogens, Division of Infection and Immunity, University College London, London, UK

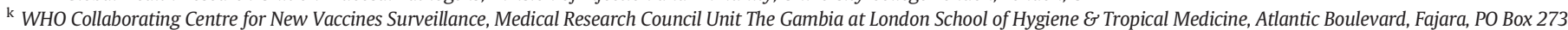
Banjul, the Gambia

${ }^{1}$ The Faculty of Health Sciences, Ben-Gurion University of the Negev, Beer-Sheva, Israel

${ }^{m}$ Medical Research Council: Respiratory and Meningeal Pathogens Research Unit, University of the Witwatersrand, South Africa

${ }^{n}$ Department of Science and Technology, National Research Foundation: Vaccine Preventable Diseases, University of the Witwatersrand, South Africa

${ }^{\circ}$ Rollins School Public Health, Emory University, USA

p Queens Research Institute, University of Edinburgh, UK

${ }^{\mathrm{q}}$ Division of Microbiology \&'Immunity, Warwick Medical School, University of Warwick, Coventry, CV4 7AL, UK

${ }^{\mathrm{r}}$ Centers for Disease Control and Prevention, Atlanta, USA

${ }^{s}$ Emory Global Health Institute, Atlanta, USA

\section{A R T I C L E I N F O}

\section{Article history:}

Received 11 March 2019

Received in revised form 5 April 2019

Accepted 9 April 2019

Available online 16 April 2019

\begin{abstract}
A B S T R A C T
Background: Pneumococcal conjugate vaccines have reduced the incidence of invasive pneumococcal disease, caused by vaccine serotypes, but non-vaccine-serotypes remain a concern. We used whole genome sequencing to study pneumococcal serotype, antibiotic resistance and invasiveness, in the context of genetic background. Methods: Our dataset of 13,454 genomes, combined with four published genomic datasets, represented Africa (40\%), Asia (25\%), Europe (19\%), North America (12\%), and South America (5\%). These 20,027 pneumococcal genomes were clustered into lineages using PopPUNK, and named Global Pneumococcal Sequence Clusters (GPSCs). From our dataset, we additionally derived serotype and sequence type, and predicted antibiotic sensitivity. We then measured invasiveness using odds ratios that relating prevalence in invasive pneumococcal disease to carriage.

Findings: The combined collections $(n=20,027)$ were clustered into 621 GPSCs. Thirty-five GPSCs observed in our dataset were represented by $>100$ isolates, and subsequently classed as dominant-GPSCs. In 22/35 (63\%) of dominant-GPSCs both non-vaccine serotypes and vaccine serotypes were observed in the years up until, and including, the first year of pneumococcal conjugate vaccine introduction.

Penicillin and multidrug resistance were higher $(p<.05)$ in a subset dominant-GPSCs (14/35, 9/35 respectively), and resistance to an increasing number of antibiotic classes was associated with increased recombination $\left(\mathrm{R}^{2}=\right.$ $0.27 p<.0001)$. In $28 / 35$ dominant-GPSCs, the country of isolation was a significant predictor $(p<.05)$ of its antibiogram (mean misclassification error $0.28, \mathrm{SD} \pm 0.13$ ).
\end{abstract}

\footnotetext{
* Corresponding author.

E-mail address: rg9@sanger.ac.uk (R.A. Gladstone).

1 These authors contributed equally

2 http://www.pneumogen.net/gps/
} 
We detected increased invasiveness of six genetic backgrounds, when compared to other genetic backgrounds expressing the same serotype. Up to 1.6-fold changes in invasiveness odds ratio were observed.

Interpretation: We define GPSCs that can be assigned to any pneumococcal genomic dataset, to aid international comparisons. Existing non-vaccine-serotypes in most GPSCs preclude the removal of these lineages by pneumococcal conjugate vaccines; leaving potential for serotype replacement. A subset of GPSCs have increased resistance, and/or serotype-independent invasiveness.

(c) 2019 The Authors. Published by Elsevier B.V. This is an open access article under the CC BY license (http:// creativecommons.org/licenses/by/4.0/).

\section{Research in context}

\section{Evidence before this study}

We searched PubMed using the terms "Streptococcus pneumoniae" OR "pneumococcus" AND "genome sequencing" OR "invasiveness disease potential" AND "genotype" OR "clone", for papers published in English between Jan 1, 2000, and Aug 21, 2018. Whole genome sequencing of pneumococci has mainly been used for detailed characterisation of strains or lineages. While pneumococcal population structure influences pneumococcal conjugate vaccine impact, only a small number of in-depth descriptions have been performed using species-wide genomic surveys of carriage or disease: Maela, Thailand [3085 isolates], Southampton UK [672 isolates], Massachusetts US [616 isolates], Blantyre, Malawi [585 isolates], Nijmegen, The Netherlands [346 isolates]. These studies all used Bayesian Analysis of Population Structure, which provides robust but dataset specific clustering. Publications over the last 15 years have periodically indicated that genotype influences invasiveness, for some pneumococcal lineages, but this subject has received little attention relative to serotype.

\section{Added value of this study}

An international genome-based scheme for defining pneumococcal population structure, allowed us to characterise and compare lineages across countries, giving international context to serotype, antibiotic resistance and geographical spread. This study uses GPSC definition of genotype to measure invasiveness, adding to the evidence that genotype can influence invasiveness.

\section{Implications of all the available evidence}

An international definition of pneumococcal population structure, unifies current and future genomic collections, facilitating comparisons. Increasing knowledge of geographical spread, distribution of antibiotic resistance, existence of non-vaccine-type variants and the invasive contribution of genotypes, provides useful context for assessing PCV impact. The generated genomic data offers a considerable resource, to further investigate the biology behind key themes in global control of pneumococcal disease.

\section{Background}

Pneumococcal conjugate vaccines (PCVs) are highly effective in the prevention of invasive pneumococcal disease, caused by vaccine serotypes [1]. PCVs with seven, 10 or 13 conjugated serotypes are in use in $\sim 150$ countries [2]. Further conjugate vaccines are in development including PCV15 (Merck) [3] and a 20-valent formulation (Pfizer) [4], both in phase III clinical trials. Young children are the main carriers of
Streptococcus pneumoniae, and immunisation of this age group protects them against invasive pneumococcal disease (IPD), caused by vaccine serotypes. Replacement of vaccine serotypes in carriage and disease by non-vaccine serotypes, termed serotype replacement, has offset some of the disease reductions in vaccinated and unvaccinated age groups $[5,6]$. The pneumococcal capsular polysaccharides are used to classify the pneumococcus into 100 serotypes based on antibody binding to specific epitopes. Serotype is considered the primary pneumococcal virulence determinant [7].

Pneumococcal population structure can be characterised using multi-locus sequence typing (MLST), that determines the sequence variation in seven housekeeping genes. At least one MLST gene $(d d l)$ has been linked to a known recombination hotspot in pneumococci [8]. MLST is limited in its ability to infer relationships between all strains [9], as shared ancestry can be masked by recombination and variation that has accumulated over longer timescales. Whole genome sequencing has increased resolution, allowing relationships between strains across the species to be established. Public health bodies are now taking steps to integrate pneumococcal whole genome sequencing into routine microbiology. Public Health England and the Centers for Disease Control and Prevention, have published methods using pneumococcal whole genome sequencing for determining serotype, and predicting antibiotic susceptibility $[10,11]$.

Multiple studies have used pneumococcal genomics to investigate PCV impact [12-15]. They often cluster the population into groups using genomic variation that reflects a shared evolutionary history. To date, these genomic definitions of lineages have been dataset-specific, unlike MLST, hindering their use when comparing studies. The Global Pneumococcal Sequencing project (GPS, http://www.pneumogen.net/ gps/), aims to provide an international understanding of pneumococcal population structure and PCV impact. It includes pneumococcal collections from invasive disease, and asymptomatic carriage. Multiple lowand middle-income countries are represented, and where possible, samples collected before and after PCV introductions. We aimed to use genome-wide variation to capture signals of shared descent, and define Global Pneumococcal Sequence Clusters (GPSCs). We used the GPSCs to provide further context on the distribution of serotypes, antibiotic resistance and invasiveness across pneumococcal lineages, which can aid assessments of PCV-impact [16,17].

\section{Methods}

\subsection{Study design}

We included 13,454 pneumococcal genomes available from the ongoing GPS project by June 2017. Investigators from each country provided epidemiological information including clinical manifestation, host age group, isolation year and sample source (Supplementary T1). IPD isolates were from a normally sterile site, while carriage isolates were from nasopharyngeal or nasal swabs. Participating laboratories performed antibiotic susceptibility testing where facilities allowed. We interpreted the susceptibility data as SIR (susceptible, intermediate, resistant) using Clinical Laboratory Standard Institute (CLSI) M100ED28:2018. We applied the meningitis threshold for penicillin on all isolates, to allow assessment and comparison of penicillin resistance 
between GPSCs. Phenotypic antibiotic susceptibility data were available for $<50 \%$ of isolates. This available data could be used to assess the sensitivity and specificity of genotypic prediction, and the validity of generating new, genome-derived, susceptibility data for several countries. The pre-PCV period was defined as the years when no conjugate vaccine was used and the year of first PCV introduction in each country.

\subsection{Whole genome analysis}

The following whole genome analyses are expanded in Supplementary Methods. Briefly, isolates were Illumina sequenced and raw data, assembled [18] and deposited in the European Nucleotide Archive (Supplementary T1). We derived MLST sequences types (STs) and serotype from the genome $[19,20]$. We further screened for the presence of known resistance conferring genes and mutations - for penicillin, tetracycline, erythromycin, chloramphenicol, co-trimoxazole - in the CDC pneumococcal typing pipeline database $[11,21,22]$. References to serotype and resistance throughout are from genomic inference. Multidrug resistance was defined as predicted resistance to $\geq 3$ antibiotic classes.

To define GPSCs and improve global representation, the GPS dataset ( $n=13,454)$ was supplemented with published datasets from the Netherlands ( $n=2803)$, Thailand $(n=2663)$, USA $(n=616)$ and UK $(n=491)$ (Supplementary T2). We used PopPUNK to group isolates from this combined dataset $(n=20027)$ into lineages, which clusters them using core and accessory distances (Fig. S1) [23]. We coined these lineages Global Pneumococcal Sequence Clusters (GPSCs), and created a reference database - available at https://www.pneumogen. net/gps/assigningGPSCs.html - that can be used with popPUNK to assign the GPSCs to new data. HierBAPS was used as a clustering comparator [24]. It was run on a SNP alignment generated by mapping GPS isolates to ATCC 700669. Recombination was quantified among core genes using FastGEAR on a representative set of STs (Supplementary T3). Recombination within each dominant-GPSC was quantified using Gubbins after mapping to a GPSC specific reference (Supplementary T4) $[25,26]$. Pairwise SNP distances were calculated for a core gene alignment of the GPS dataset generated via Roary [27], and for recombination-free alignments per dominant-GPSC, using the Pairsnp-r R package.

\subsection{Statistical analysis}

Estimates of the number of GPSCs in the true population were modelled using the R Vegan package [28]. Simpson's Diversity index 1-D (SDI) reports no diversity (zero) to unlimited diversity (one). We tested the predictive value of dominant-GPSCs, for antibiogram or serotype. We then tested the predictive value of country, for antibiogram or serotype, within each dominant-GPSC. We compared multinomial logistic regressions to null models using a likelihood ratio test. Input data was restricted to the un-perturbed pre-PCV population. Corrections for multiple testing $(n \geq 10)$ used the Benjamini-Hochberg false discovery rate of $5 \%$. Pneumococcal heritability $\left(h^{2}\right)$ of invasiveness was calculated with a regression of all genomic variation using Pyseer [29] on South African isolates, from children $<7$ years of age in which variation in invasiveness is limited (Supplementary T5) [30]. The explanatory value of serotype for invasiveness was measured using Nagelkerke's pseudo- $\mathrm{R}^{2}$ [31]. Quantification of invasiveness was performed using odds-ratios (OR) for invasive disease where prevalence in invasive disease was related to their prevalence in carriage. We performed an OR meta-analysis of data from South Africa (national IPD $n$ $=625$, carriage; Agincourt $n=798$, Soweto $n=291$ ) and USA (national IPD $n=456$, carriage Massachusetts $n=345$ ), using individuals $<7$ years old (Supplementary T6). The Cochran's Q-test was used to detect heterogeneity by country within estimated ORs. The (log) odds ratio for invasive disease used Peto's method where $a$ is the number of invasive isolates of $\mathrm{X}$, where $\mathrm{X}$ denotes a particular serotype, genotype or serotype-genotype, $b$ is the number of carriage isolates of $\mathrm{X}, c$ is the number of non-X invasive isolates, and $d$ is the number of non-X carriage isolates, in line with previous work by Brueggemann et al [7]. Measuring differences in the proportion of IPD cases to carriage were performed between the pairs of most and least invasive genotypes, using the OR dataset from the country (USA or South Africa) they were predominantly observed. These statistical analyses are expanded in Supplementary Methods.

\section{Findings}

Our GPS dataset included 13,454 isolates representing 30 countries, and 5 continents: Africa (13 countries, 59\% of collection), Asia (8, 18\%), South America (2, 8\%), Europe (4, 3\%) and North America (3, 12\%). GPS key countries ( $n>1000)$ included South Africa ( $n=4615,63 \%$ IPD), The Gambia ( $n=1647,24 \%$ IPD), Malawi ( $n=1304,43 \%$ IPD), Israel ( $n=$ $1143,100 \%$ IPD) and USA ( $n=1584,100 \%$ IPD). Sixty-four percent of the collection were isolated from IPD, $96 \%$ of the collection were isolated between 2000 and 2017 and 74\% were from children aged $\leq 5$ years old (Table 1, Fig. S2).

Genome-wide variation in our dataset combined with published collections (total $n=20,027$ ) clustered isolates into 621 GPSCs (Fig. S3). Our 621 GPSCs represent over $61 \%$ of the 1012.7 GPSCs (SE \pm 76 ) estimated to be in the true population. However, most GPSCs (407 of 621, $66 \%$ ) were rare lineages with $<10$ isolates, together representing 1043 of 20,027 (5\%) of the combined collection. Within the GPS dataset, we observed 538 GPSCs. 35 GPSCs had $>100$ isolates in the GPS dataset and were classified as dominant-GPSCs. Together they represented 8356 of 13,454 (62\%) of the GPS dataset, and 5978 of 8605 (69\%) of the GPS dataset disease isolates (Fig. S4). Sampling multiple countries detected significantly more GPSCs $(p<.0001)$ than equivalent sampling from a single location (Fig. S5).

We defined MLST Clonal Complexes (CC) as STs with single locus variant (SLV) differences, within the GPS dataset. GPSCs often encompassed related CCs, with a mean number of 2.6 CCs per dominant-GPSC ( $\mathrm{SD} \pm 1.5$, excluding singleton STs). GPSCs identify a shared history not captured by CC designations. CC217, CC615, CC3581 and 2 singleton STs were clustered into GPSC2, a grouping of CCs which is widely recognised as a clonal lineage that expresses serotype 1 . CCs captured phylogenetic sub-structure well in dominantGPSCs with more than 1 CC $(n=25$, mean Consistency Index 0.9864 $\mathrm{SD} \pm 0.04$, mean Retention Index $0.9942 \mathrm{SD} \pm 0.02$ ). Our clustering additionally revealed shared descent of CC53, CC1012, CC62 and CC100 within GPSC3, that shared 0-5 MLST alleles. HierBAPS supported the clustering of $28 / 35$ (80\%) dominant-GPSCs, including GPSC3. The species-wide, core genome, pairwise SNP distances between GPSCs and within GPSCs, were predominantly non-overlapping (Fig. 1). The mean pairwise SNP distances from recombination free alignments, were broadly comparable between dominant-GPSCs, though eight GPSCs had SNPs distances of $>500$ for a subset of their isolates (Fig. 2). HierBAPS supported half of those clusters with $>500$ SNP distances, but GPSC18, GPSC23, GPSC37 and GPSC41 were split into two subclusters. Conversely GPSC1, the clonal serotype one lineage GPSC2 and GPSC16 were split by HierBAPS into two sub-clusters even when the maximum SNP distances were $<500$.

MLST genes gki, gdh, recP, spi and $d d l$ ranked in the top 6-22\% of the 1193 core genes, for recombination frequency (Supplementary T7). Disruption of vertical inheritance may result in isolates that are missed by CC: within the dominant-GPSCs 370 of 8356 isolates (4.4\%), belonging to 165 STs, were not assigned to a CC. Conversely recombination can result in convergent MLST profiles in disparate isolates, and CC designation using large collections are more vulnerable to spurious connections. Sixteen CCs spanned multiple related GPSCs and 24 isolates were assigned to 6 CCs highly discordant with their core genome phylogenetic relationship (Fig. S6).

Geographical diversity varied considerably per GPSC (Fig. 3A), though all dominant-GPSCs were observed in more than one country. 
Table 1

Clinical characteristics, age group, sex, clinical manifest and sample source, PCV period.

\begin{tabular}{lllllllll}
\hline Category & $\leq 2$ & $3-5$ & $6-15$ & $16-24$ & $25-44$ & $45-65$ & $>65$ & Total \\
\hline $\begin{array}{l}\text { Total } \\
\text { Sex }\end{array}$ & 7179 & 2790 & 1061 & 398 & 982 & 602 & 442 & 13,454 \\
F & & & & & & & & \\
M & 2863 & 972 & 440 & 214 & 502 & 173 & 89 & 5253 \\
Missing & 3288 & 1067 & 486 & 150 & 343 & 209 & 117 & 5660 \\
Manifest & 1028 & 751 & 135 & 34 & 137 & 220 & 236 & 2541 \\
$\quad$ Carriage & & & & & & & & \\
Disease & 2841 & 935 & 646 & 223 & 173 & 25 & 6 & 4849 \\
Source & 4338 & 1855 & 415 & 175 & 809 & 577 & 436 & 8605 \\
Blood & & & & & & & & \\
CSF & 2998 & 1234 & 256 & 93 & 471 & 404 & 332 & 5788 \\
Nasopharynx & 1070 & 444 & 135 & 67 & 290 & 132 & 55 & 2193 \\
Other disease & 2841 & 934 & 646 & 223 & 173 & 25 & 6 & 4848 \\
$\quad$ (non-invasive) & 256 & 173 & 24 & 15 & 45 & 38 & 46 & 597 \\
Missing & & & & & & & & \\
PCV period & 14 & 5 & 0 & 0 & 3 & 3 & 3 & 28 \\
Pre-PCV & & & & & & & & \\
Post-PCV7 & 3925 & 1439 & 671 & 250 & 418 & 230 & 177 & 7110 \\
Post-PCV10 & 1355 & 547 & 153 & 52 & 211 & 143 & 113 & 2574 \\
Post-PCV13 & 131 & 44 & 13 & 7 & 22 & 44 & 12 & 273 \\
No universal & 1592 & 671 & 210 & 86 & 314 & 177 & 131 & 3181 \\
$\quad$ introduction of & 176 & 89 & 14 & 3 & 17 & 8 & 9 & 316 \\
$\quad$ PCV & & & & & & & & \\
\hline
\end{tabular}

Isolates were classified by PCV use in the country and year of their isolation. The pre-PCV period was defined as the years when no conjugate vaccine was used and the year of first PCV introduction, the post-PCV7 period from the second year of PCV7 introduction through to the first year of PCV10 introduction, the post-PCV10 period from the second year of PCV10 introduction through to the first year of PCV13 introduction and the postPCV13 period from the second year of PCV13 introduction through to the last collection year. No universal introduction of PCV denotes years in which a nationwide immunisation policy did not exist.

Eight of 35 (23\%) had high geographical diversity (SDI >0.70) representing even distribution across 5 continents (Supplementary T8). Conversely, seven of 35 (20\%) dominant-GPSCs were observed only in Africa.

Genomic inference of serotype was reliable with 97\% (95\% CI 97.15-97.75\%) serotype co-concordance with available phenotypic data ( $n=10,466$, Supplementary T9). Of 35 dominant-GPSCs, 30 (86\%) were observed with more than one serotype. Dominant-GPSC was a significant predictor of serotype with a mean misclassification error of 0.18 , although serotype diversity within dominant-GPSCs varied considerably $(p<.0001)$, Fig. 3B). Country was a significant predictor of serotype within 18 of 35 dominant-GPSCs (51\%) with a mean misclassification error of 0.19 ( $S D \pm 0.14, p<.05$ ). For example, in GPSC3, serotype 8 was expressed in 113 of 148 (76\%) IPD isolates from South Africa and all were CC53, conversely, serotype 33F was expressed in 49 of 87 IPD isolates from the USA and 44 of 49 (90\%) were CC100/ST2705.

Two of the 35 dominant-GPSC (6\%, GPSC14, GPSC37) included isolates exclusively expressing PCV7-serotypes pre-PCV and therefore were completely covered by PCV7. However, GPSC14 and 37 accounted for only 169 of 4221 (4\%) of pre-PCV disease isolates. The number of dominant-GPSCs expressing only VTs pre-PCV, increased to 6 GPSCs for PCV10 (885 of 4221 [21\%] of pre-PCV IPD isolates), and 11 GPSCs for PCV13 (1234 of 4221 [29\% pre-PCV IPD]; Fig. 4A). The experimental 15 -valent vaccine offered no additional dominant-GPSCs expressing only VTs. The putative 20-valent formulation meant that a further four dominant-GPSCs were expressing only VTs pre-PCV (1592 of [37\%] of pre-PCV IPD isolates).

Of the 35 dominant-GPSCs, 22 (63\%) expressed non-vaccine serotypes (NVT) alongside PCV13 serotypes prior to PCV introductions. Though there were wide variations in the ratio of VT:NVT expressed (Fig. 4B). Of the 22 GPSCs, 12 (55\%) predominantly expressed VTs. NVT-variants were not observed in all locations, in South Africa, 17 of 34 (50\%) GPSCs with 10 or more isolates pre-PCV $(n=1534)$, expressed both PCV13 and NVTs pre-PCV. There were two dominant and 60 intermediate GPSCs expressing only NVTs, respectively they accounted for 20 of 4221 (0.5\%) and 208 of 4221 (5\%) of IPD isolates pre-PCV.

The positive predictive values for genetic determinants of resistance to penicillin, tetracycline, erythromycin, chloramphenicol, and cotrimoxazole were all >90\% (95\%CI 90-98.7\%, Supplementary T10). Resistance to at least one antibiotic class was predicted for 8241 of 13,454 isolates in the GPS dataset (61\%; Fig. S7). The percentage of isolates predicted to be resistant per class was not uniform across dominant-GPSCs (Fig. 5). The predicted resistance profile of an isolate could be predicted by which dominant-GPSC it belonged to half of the time, ( $p<.0001$, misclassification error 0.49). Generally, higher recombination ratios ( $\mathrm{rho} /$ theta, $\mathrm{r} / \mathrm{m}$ ), were associated with a higher mean number of classes of predicted antibiotic resistance (rho/theta $\mathrm{R}^{2}=$ $0.27, p<.0001, \mathrm{r} / \mathrm{m} \mathrm{R}^{2}=0.22, p<.0001$, Supplementary T8 and T11). GPSC1 had an above average $\mathrm{r} / \mathrm{m}$ (8.3) and rho/theta (0.14) and a predominant predicted MDR antibiogram of penicillin, co-trimoxazole, erythromycin and tetracycline resistance, but susceptibility to chloramphenicol, occurring in 388 of 504 isolates (77\%). Although this MDR antibiogram was the most common pattern in 17 of 19 countries represented in GPSC1, country was a predictor of the antibiogram for 28 of the 35 dominant-GPSCs $(80 \% ; p<.05$, mean misclassification error $0.28, S D \pm 0.13)$. Predicted penicillin resistant isolates accounted for a higher proportion of isolates within 14/35 dominant-GPSCs than expected given proportion of predicted penicillin resistance in the rest of the GPS dataset ( $p<.05,63-100 \%$, Supplementary T12). Predicted multidrug resistant isolates, accounted for higher proportion of isolates within $9 / 35$ dominant-GPSCs than expected ( $p<.05,45-77 \%$ ). Eight of these were GPSCs also found to have a higher proportion of isolates penicillin resistant isolates (Supplementary T13). Prior to PCV introductions, penicillin resistance was predicted to occurred in 2133 of 4975 (43\%) of the isolates expressing PCV13 VTs and in only 256 of 2135 (12\%) of the NVT expressing strains $(p<.0001)$.

In 9 of the 22 (40\%) GPSCs expressing both VT and NVT, the NVT component had a significantly lower proportion of predicted resistant isolates than their VT counterparts (Supplementary T14). Seven intermediate GPSCs expressing only NVTs had $>90 \%$ of isolates predicted resistant to penicillin (GPSC55, 89 of 90, [99\%]; GPSC59, 37/37 of [100\%]; GPSC81, 35 of 38 [92\%]; GPSC132, 17 of 17, 100\%]; GPSC136, 19 of 21 [90\%]; GPSC168, 15 of 15 [100\%]; GPSC200, 11 of 11 [100\%]).

In the South African heritability dataset, serotype explained a third of the strain variation in clinical manifestation (carriage or disease, pseudo- $R^{2}$ 0.32). Total pneumococcal genetic variation (including the cps locus which encodes the CPS) was a better explanation $\left(h^{2} 0.57\right)$, explaining over half of the variation in clinical manifestation, leaving some invasiveness explained by genes outside the cps locus.

The $95 \% \mathrm{CI}$, for invasiveness ORs did not overlap between at least one pair of genotypes (GPSC $n=96$, ST $n=112$ ) within serotypes 6A, 14, 16F, 19F, 23B and 23F (Table 2, Fig. S8). Only within GPSC14 was a genotype with increased invasiveness found to be significant at the ST level but not the GPSC level, as ST6279 and ST2059, both found within GPSC14, significantly differed in invasiveness (Table 2, Supplementary $\mathrm{T} 15$ and T16). We detected significant heterogeneity in invasiveness for serotype 38-GPSC38/ST393 ( $\mathrm{Q}=3.877$ [df 1 ], $p<.05$ ), between South Africa (OR 0.67, 95\%CI 0.24-1.88) and the USA (OR 6.83, 95\%CI 0.86-54.20) in the meta-analysis estimate of the OR. Despite the small sample numbers, the two countries significantly differed in the proportion of serotype 38 -GPSC38 from IPD $(p=.008)$. A conservative comparison using the upper $\mathrm{CI}$ of the least invasive genotype and the lower $\mathrm{CI}$ of the most invasive genotype had 1.05 to 1.6-fold changes in OR (Table 2). The influence of genotype on invasiveness can be of a similar magnitude to some serotypes. For example a 1.6-fold change was observed between the upper $\mathrm{CI}$ of the less invasive serotype 35A $(0.12$ [0.016-0.938], $p=.043$ ) and the lower $\mathrm{Cl}$ of the more invasive serotype 18C (3.237[1.514-6.921], $p=.0024)$ determined in this dataset (Supplementary T17). 


\section{Pairwise core SNP distances between and within GPSCs}

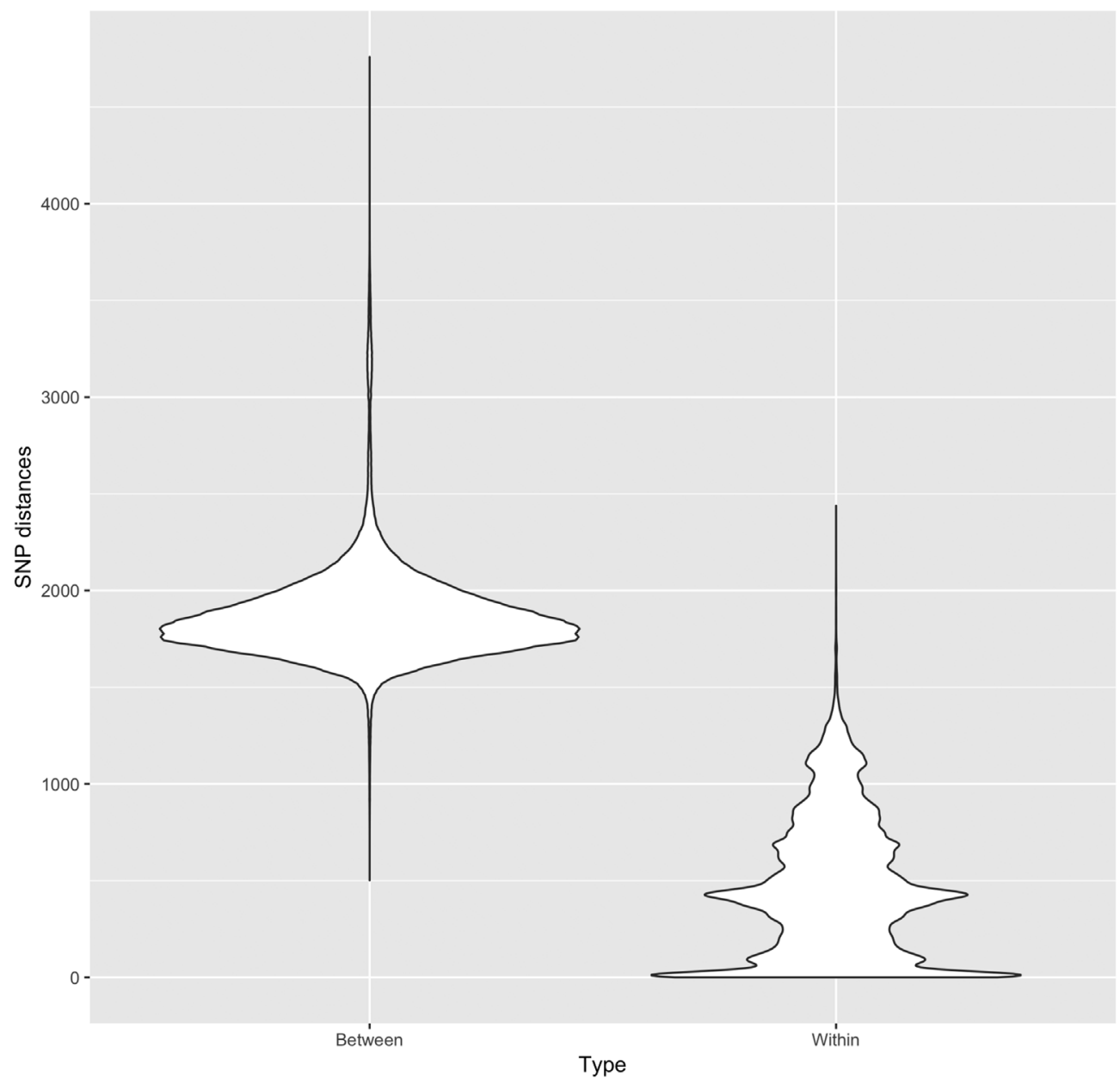

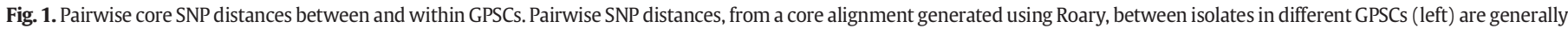
greater than pairwise SNP distances for isolates within the same GPSC (Right).

\section{Discussion}

We present the distribution of key themes in pneumococcal disease control, such as serotype, antibiotic resistance and invasiveness, in a large international collection. We used genome-wide variation to define Global Pneumococcal Sequence Clusters (GPSCs), to produce a dataset independent genomic definition of lineages. Increasing knowledge of the spread of traits across the pneumococcal population and geographical regions, gives greater context for assessing the impact of PCV introduction.
At an international level, we have shown that pneumococcal nonvaccine serotypes exist alongside vaccine serotypes, within dominant GPSCs that account for the majority of the pneumococcal population. The existence of non-vaccine serotype variants negates reliance on contemporaneous capsule switch events for "vaccine escape" of a GPSC. Given that the pneumococcus has multiple lineages that are globally disseminated there is potential for non-vaccine types established in one geographical region to spread globally, or be present but undetected in other countries [32]. Indeed, previous carriage studies have observed the importation of lineages not previously observed in that

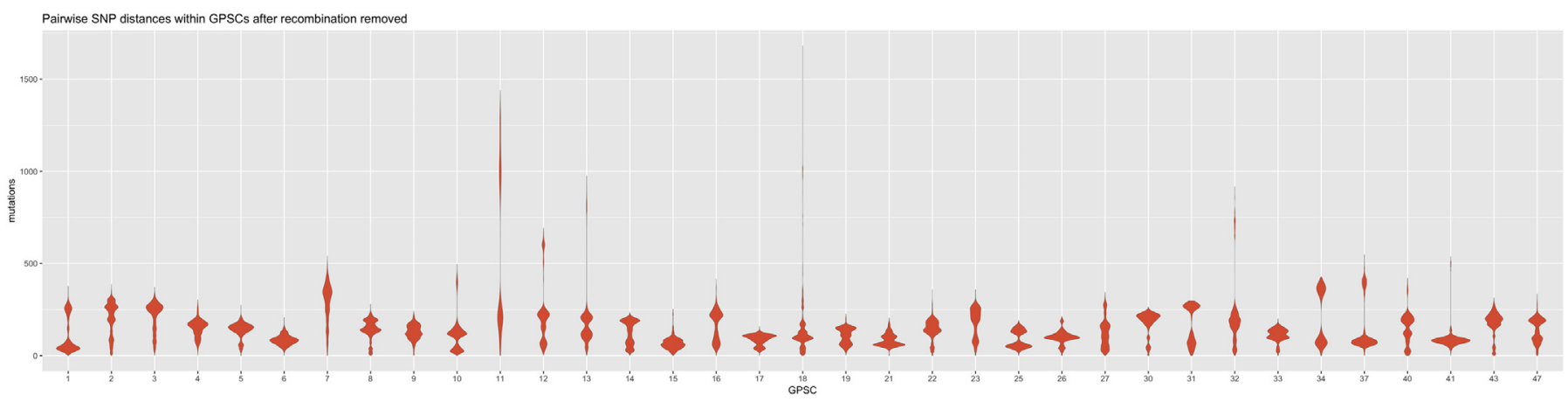

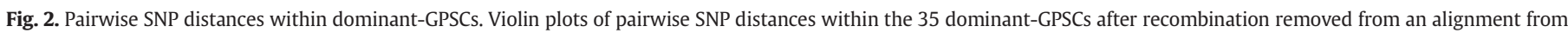
mapping to an internal reference for each GPSC are largely comparable. 
a

क

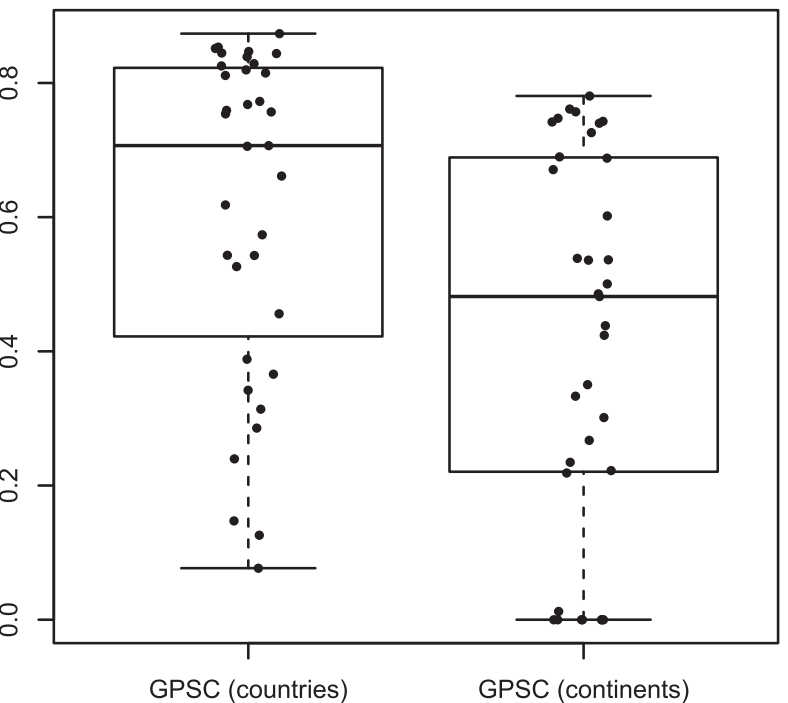

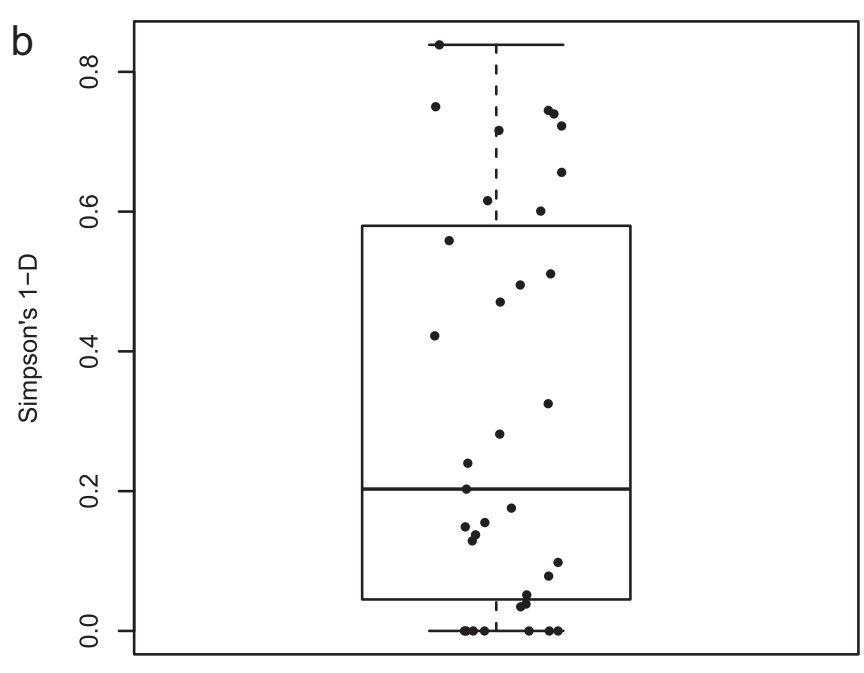

GPSC (serotypes)

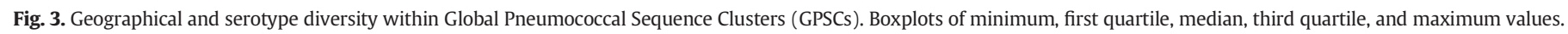

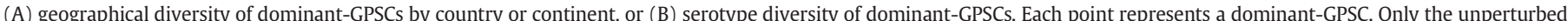

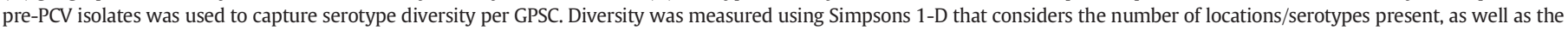
relative abundance of each location/serotype. Zero denotes no diversity and 1 denotes unlimited diversity.

location, and further estimated that the influx of new lineages would result in a 50\% population turnover after 13 years [16,33].

We showed that antibiotic resistance was enriched in a subset of GPSCs, many of which were dominant and globally disseminated. We observed that both GPSC, and country within GPSCs were significant predictors of the antibiotic resistance pattern of an isolate. GPSCs with an increased propensity for resistance, whether associated with increased capacity for recombination, duration of carriage [34], can spread to other locations. Loss of resistance, in the absence of selection, has been reported for lineages in countries with lower antibiotic prescription rates; multiple independent losses of resistance to chloramphenicol, tetracycline and erythromycin were observed for Pneumococcal Molecular Epidemiology Network (PMEN)2 in Iceland [35]. However, over a decade after a reduction in antibiotic consumption, the majority

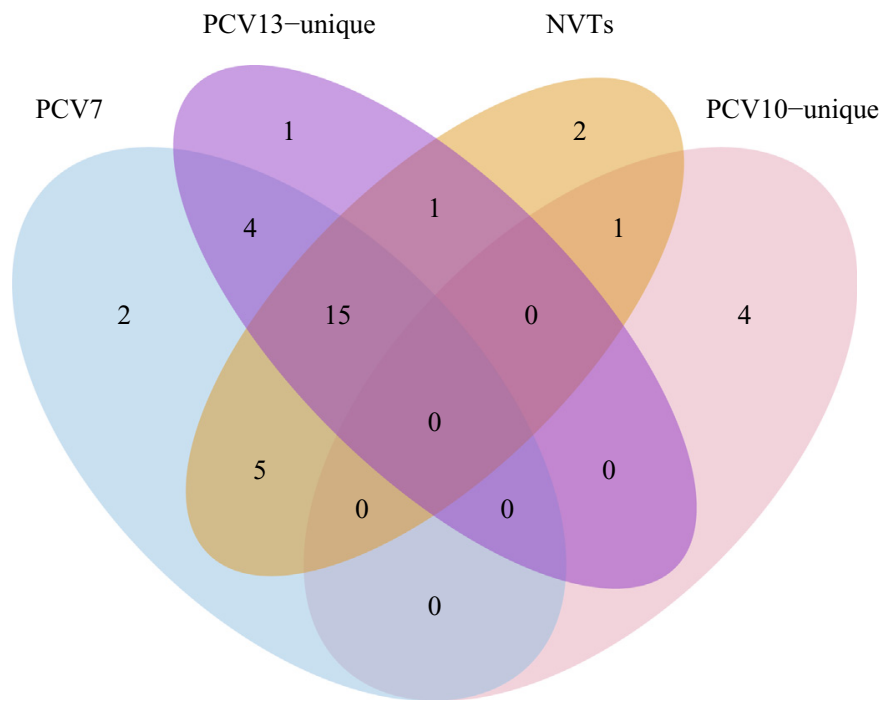

of Icelandic PMEN2 remained resistant, albeit at a reduced prevalence, and as such lineages remain a risk to high usage settings. Antibiotic resistance is lower in non-vaccine serotypes, but this prevalence varies substantially by GPSC. Some notable GPSCs expressing only nonvaccine serotypes do have high levels of penicillin resistance, and within GPSCs that express both non-vaccine and vaccine serotypes, the nonvaccine serotypes occasionally have similar or higher resistance profiles to their vaccine serotype counterparts.

Preservation of gene frequencies in the population through negative frequency-dependent selection has been shown to exist in pneumococci, and can be used to predict serotype replacement in carriage $[16,36]$. This suggests that the gene content of a GPSCs influences whether it will undergo replacement or expansion after vaccine perturbation. Only genomic data combined with a robust clustering method

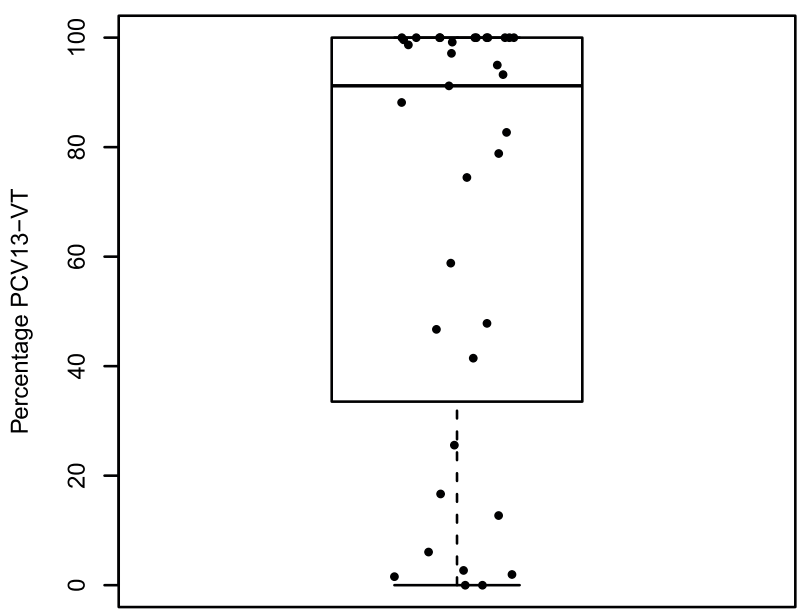

Dominant-GPSC

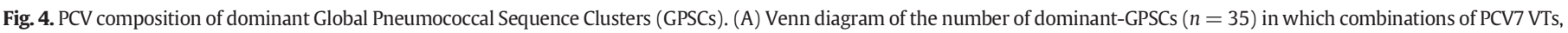

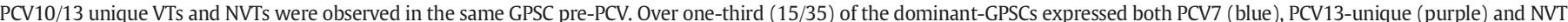

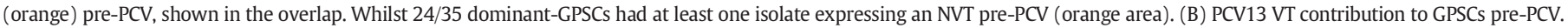

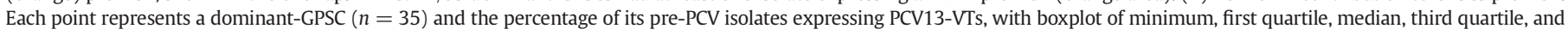
maximum values. 


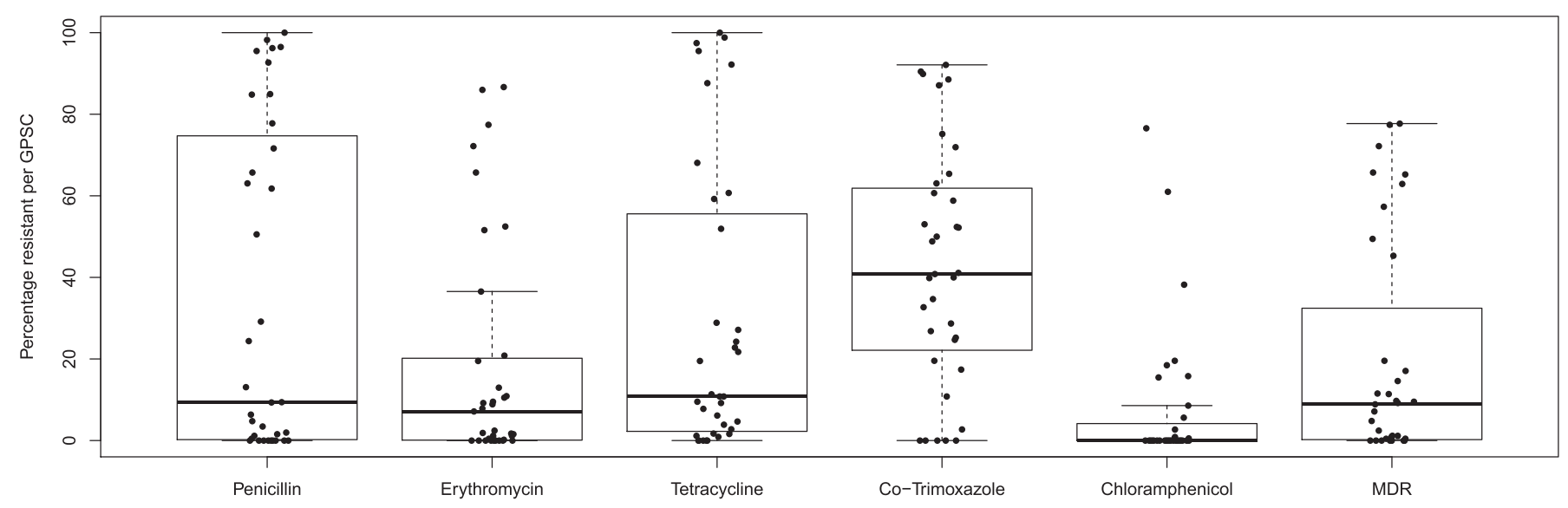

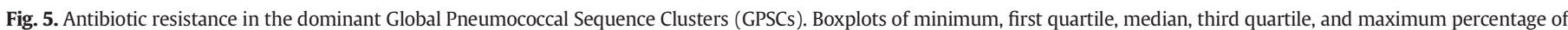

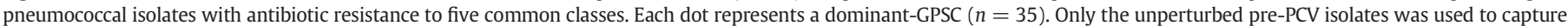
the distribution of resistance to each class across the dominant-GPSCs.

has the power to model such complex dynamics. Non-vaccine and vaccine serotypes within the same GPSC, will share similar gene complements and ecological phenotypes, including resistance. Non-vaccine variants may therefore have increased potential to replace their vaccine-type counterparts compared to other GPSCs expressing nonvaccine serotypes $[13,37]$. The GPSCs involved in any replacement will determine the extent to which reductions, not only in total disease, but in antibiotic resistance, could be partially offset by non-vaccine serotypes. This has been observed with pneumococcal serotype replacement after routine use of the 7-valent conjugate vaccine by multidrug resistant 19A in the US within GPSC1(CC320), which slowed reductions in total disease, antibiotic resistance, and subsequently, the cost effectiveness of PCV7 [38].

Serotype is a potent virulence determinant, however other virulence factors exist in the genome outside of the cps locus. Genotypes have previously been implicated in invasiveness in a number of small studies using MLST/pulsed-field gel electrophoresis definitions, some of which are complicated by age-related differences in invasiveness [30,39-41]. With a substantial collection of pneumococcal genomes, we have used heritability analysis and difference in invasiveness ORs to demonstrate that genome variation beyond serotype contributes to invasiveness in children $<7$ years old. Measurable differences in invasiveness ORs between individual GPSCs and STs were comparable to a change in serotype. Stratification of serotype by genotype (GPSC or ST) significantly impacts sample size and subsequent power to detect subtle influences of genotype. Many serotypes were not represented by multiple genotypes in our dataset preventing the contribution of those GPSCs being fully assessed. The generation of further temporally matched and genotyped collections from carriage and disease would increase power and allow further investigation of our findings. Comparing national surveillance with local carriage has the potential to introduce bias, but with sufficient sampling acts as a convenient proxy for the national population. Despite these limitations, differences in invasiveness within serotypes was observed in nearly a quarter $(23 \%, 6 / 26)$ of the serotypes tested. Genetic determinants of invasiveness need not be uniform across a genotype; pneumococci within a given genotype will differ slightly in gene content and sequence. This fits with our observations of differences between STs within 23F GPSC14/CC6279, heterogeneity within GPSC38 between countries, and previous work by others showing differing invasiveness of PFGE clones within CC138 [41]. Our work independently observes the increased invasiveness of serotype 14 in a GPSC18/CC15 background compared to at least one other genotype, as previously reported, and we highlight differential invasiveness for genotypes within 6 serotypes in total [41].

There is substantial sequence variation in the cps locus within serotypes to acknowledge [42]. Such variation could represent undetected differences in capsular structure, which could in turn influence invasiveness. This was the case for serotype $6 \mathrm{C}$ before it was discriminated from $6 \mathrm{~A}$, though conversely, divergent genotypes of serotype $6 \mathrm{~B}$ still result in the same polysaccharide $[42,43]$. The cps loci within serotypes in the GPS collection have been investigated [44]; of the serotypes implicated as differing in invasiveness, only $16 \mathrm{~F}$ in GPSC33 has an atypical cps locus, which may explain why we observed it was less invasive than GPSC46, which has the typical $16 \mathrm{~F}$ cps genotype. After ruling out the influence of novel serotypes, identification of the genetic variation driving the observed invasiveness of GPSCs could offer alternative vaccine targets associated with severe disease. Genome wide association studies (GWAS) have been used by others to identify candidate genetic variation associated with different manifestations of disease [31,45,46].

While MLST is limited in resolution to infer strain relatedness, it is an internationally reproducible scheme. To date an international scheme has not existed for clustering pneumococcal isolates using whole genome data, and alternative methods for clustering genomic collections of this scale are scarce. Furthermore, current methods lack the ability to place a strain in an existing framework, consequently clustering an additional strain would require re-running an entire collection without

Table 2

Pneumococcal invasiveness for pairs of genotypes that significantly differed within a serotype.

\begin{tabular}{|c|c|c|c|c|c|c|}
\hline Serotype & $\begin{array}{l}\text { Least invasive } \\
\text { (predominant CC) }\end{array}$ & OR $[95 \% \mathrm{CI}]$ & $\begin{array}{l}\text { Most invasive } \\
\text { (predominant CC) }\end{array}$ & OR $[95 \% \mathrm{CI}]$ & Conservative OR fold change & $\begin{array}{l}\text { Fisher's } p \text {-value } \\
\text { (country) }\end{array}$ \\
\hline $6 \mathrm{~A}$ & GPSC5 (CC172) & $0.34[0.12-1.01]$ & GPSC41 (CC1094) & $2.96[1.61-5.45]$ & 1.6 & $0.0004(\mathrm{ZA})$ \\
\hline 14 & GPSC9 (CC63) & $0.62[0.22-1.73]$ & GPSC18 (CC15) & $12.45[2.82-54.98]$ & 1.6 & $0.0005(\mathrm{ZA})$ \\
\hline $16 \mathrm{~F}$ & GPSC33 (CC4088) & $0.14[0.06-0.36]$ & GPSC46 (CC30) & $2.62[0.44-15.73]$ & 1.2 & $0.0099(\mathrm{ZA})$ \\
\hline $19 \mathrm{~F}$ & GPSC21 (CC347) & $0.32[0.19-0.54]$ & GPSC1 (CC320) & $1.49[0.78-2.88]$ & 1.4 & $0.0006(\mathrm{ZA})$ \\
\hline 23B & GPSC7 (CC439) & $0.14[0.05-0.42]$ & GPSC5 (CC172) & $3.81[0.44-32.79]$ & 1.05 & 0.005 (USA) \\
\hline $23 \mathrm{~F}$ & GPSC14 ST6279 (CC6279) & $0.81[0.43-1.54]$ & GPSC14 ST2059 (CC6279) & $5.31[1.71-16.54]$ & 1.1 & $0.004(\mathrm{ZA})$ \\
\hline
\end{tabular}

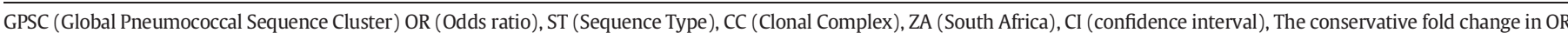
was calculated by dividing the lower $\mathrm{CI}$ of the most invasive genotype by the upper $\mathrm{CI}$ of the least invasive genotype, within each serotype. 
a reproducible clustering nomenclature. Our definition of the pneumococcal lineages (GPSCs) on an internationally sampled population, can be used to assign GPSCs to any collection of pneumococcal genomes using our database of GPSC reference genomes and PopPUNK [23]. The GPSC database can be updated when novel GPSCs are assigned in future collections, enabling stable international comparisons of pneumococcal population structure [15]. The GPSCs are broadly back-compatible with MLST as the vast majority of STs were found exclusively within a GPSC. To that end, we provide a ST-GPSC conversion table with noted exceptions, to facilitate cross referencing of non-genomic datasets (Supplementary T18). We have used these GPSC designations, genome-derived serotype and antibiotic resistance to facilitate an in depth assessment of the lineages causing invasive disease in young children in the post-PCV13 era [17], and to explore the mechanisms driving the progression of serotype replacement.

Understanding the underlying genetic variation and characteristics of GPSCs that influence resistance, invasiveness and pneumococcal population dynamics in a global context is highly informative. Such information can be used for modelling vaccine replacement, predicting vaccine impact and rational vaccine design. Our high-resolution genomic approach for defining pneumococcal lineages across different collections, in a manner that reflects pneumococcal biology, increases the evidence required to build a global strategy for continued control of pneumococcal disease.

\section{Acknowledgements}

We would like to thank all members of the GPS consortium for their collaborative spirit and determination during the monumental task of sampling, extracting and sequencing this dataset, and all contributions to experimental design and input into this manuscript. We also would like to thank members of teams 284 and 81 at the Wellcome Sanger Institute (WSI) for their advice and critique and the pathogen informatics team at the WSI for the pipelines and expertise that made genomic analysis at this scale possible. We would like to thank Diederik van de Beek and Phillip Kremer for their critical review and clinical perspective on the manuscript.

\section{Funding}

This study was co-funded by the Bill and Melinda Gates Foundation, the Wellcome Sanger Institute, and the US Centers for Disease Control and Prevention.

\section{Role of funding sources}

This study was co-funded by the Bill and Melinda Gates Foundation (grant code OPP1034556), the Wellcome Sanger Institute (core Wellcome grants 098051 and 206194) and the US Centers for Disease Control and Prevention. The funding sources had no role in isolate selection, analysis, or data interpretation. The corresponding authors had full access to the data and are responsible for the final decision to submit for publication. Isolates included from Qatar were collected through research project supported by NPRP 6-496-3-127 from Qatar National Research Foundation (QNRF).

\section{Disclaimer}

The findings and conclusions in this report are those of the authors and do not necessarily represent the official position of the Centers for Disease Control and Prevention.

\section{Ethics statement}

Isolates for this study were selected from retrospective bacterial collections in each country participating in GPS. Appropriate approvals for use of isolates was obtained from each institution contributing isolates. No tissue material or other biological material was obtained from humans. All information regarding these isolates was anonymised.

\section{Declaration of interests}

Dr. Gladstone reports PhD studentship from Pfizer, outside the submitted work; Dr. Lees reports grants from Pfizer, outside the submitted work; Dr. Madhi reports grants from BMGF, during the conduct of the study; grants and personal fees from BMGF, grants from Pfizer, grants from GSK, grants from Sanofi, grants from BIOVAC, outside the submitted work; Dr. Dagan reports grants and personal fees from Pfizer, during the conduct of the study; grants and personal fees from MSD, personal fees from MeMed, outside the submitted work; Dr. von Gottberg reports grants and other from Pfizer, during the conduct of the study; grants and other from Sanofi, outside the submitted work; Dr. Bentley reports personal fees from Pfizer, personal fees from Merck, outside the submitted work.

\section{Appendix A. Supplementary data}

Supplementary data to this article can be found online at https://doi. org/10.1016/j.ebiom.2019.04.021.

\section{References}

[1] Andrews NJ, Waight PA, Burbidge P, Pearce E, Roalfe L, Zancolli M, et al. Serotypespecific effectiveness and correlates of protection for the 13-valent pneumococcal conjugate vaccine: a postlicensure indirect cohort study. Lancet Infect Dis 2014; 14:839-46.

[2] McIntyre PB, O'Brien KL, Greenwood B, van de Beek D. Effect of vaccines on bacterial meningitis worldwide. Lancet 2012;380:1703-11.

[3] Skinner JM, Indrawati L, Cannon J, Blue J, Winters M, Macnair J, et al. Pre-clinical evaluation of a 15-valent pneumococcal conjugate vaccine (PCV15-CRM197) in an infant-rhesus monkey immunogenicity model. Vaccine 2011;29:8870-6.

[4] Emini EA, Watson WJ, Prasad AK, Han M, Kim J-H, Gu J, et al. Immunogenic compositions comprising conjugated capsular saccharide antigens and uses thereof; 2016; 9492559.

[5] Hicks LA, Harrison LH, Flannery B, Hadler JL, Schaffner W, Craig AS, et al. Incidence of pneumococcal disease due to non-pneumococcal conjugate vaccine (PCV7) serotypes in the United States during the era of widespread PCV7 vaccination, 19982004. J Infect Dis 2007;196:1346-54.

[6] Ladhani SN, Collins S, Djennad A, Sheppard CL, Borrow R, Fry NK, et al. Rapid increase in non-vaccine serotypes causing invasive pneumococcal disease in England and Wales, 2000-17: a prospective national observational cohort study. Lancet Infect Dis 2018;18:441-51.

[7] Brueggemann AB, Griffiths DT, Meats E, Peto TE, Crook DW, Spratt BG. Clonal relationships between invasive and carriage Streptococcus pneumoniae and serotypeand clone- specific differences in invasive disease potential. J Infect Dis 2003;187: 1424-32.

[8] Enright MC, Spratt BG. Extensive variation in the ddl gene of penicillin-resistant Streptococcus pneumoniae results from a hitchhiking effect driven by the penicillinbinding protein 2b gene. Mol Biol Evol 1999;16:1687-95.

[9] Tsang AKL, Lee HH, Yiu S-M, Lau SKP, Woo PCY. Failure of phylogeny inferred from multilocus sequence typing to represent bacterial phylogeny. Sci Rep 2017;7:4536.

[10] Kapatai G, Sheppard CL, Al-Shahib A, Litt DJ, Underwood AP, Harrison TG, et al. Whole genome sequencing of Streptococcus pneumoniae: development, evaluation and verification of targets for serogroup and serotype prediction using an automated pipeline. Peer 2016;4:e2477.

[11] Metcalf BJ, Gertz Jr RE, Gladstone RA, Walker H, Sherwood LK, Jackson D, et al. Strain features and distributions in pneumococci from children with invasive disease before and after 13-valent conjugate vaccine implementation in the USA. Clin Microbiol Infect 2016;22(60) [e9-60 e29].

[12] Cremers AJ, Mobegi FM, de Jonge MI, van Hijum SA, Meis JF, Hermans PW, et al. The post-vaccine microevolution of invasive Streptococcus pneumoniae. Sci Rep 2015; 5:14952.

[13] Gladstone RA, Devine V, Jones J, Cleary D, Jefferies JM, Bentley SD, et al. Pre-vaccine serotype composition within a lineage signposts its serotype replacement - a carriage study over 7 years following pneumococcal conjugate vaccine use in the UK. Microb Genom 2017;3. https://doi.org/10.1099/mgen.0.000119.

[14] Croucher NJ, Harris SR, Fraser C, Quail MA, Burton J, van der Linden M, et al. Rapid pneumococcal evolution in response to clinical interventions. Science 2011;331: 430-4.

[15] Croucher NJ, Coupland PG, Stevenson AE, Callendrello A, Bentley SD, Hanage WP. Diversification of bacterial genome content through distinct mechanisms over different timescales. Nat Commun 2014;5:5471.

[16] Corander Jukka, Fraser Christophe, Gutmann Michael U, Arnold Brian, Hanage William P, Bentley Stephen D, et al. The role of negative frequency-dependent 
selection in vaccine-induced pneumococcal population dynamics. Nat Ecol Evol 2017;1:1950-60.

[17] Lo SW, Gladstone RA, van Tonder AJ, du Plessis M, Benisty R, Givon-Lavi N, et al. Pneumococcal lineages associated with serotype replacement and antibiotic resistance in childhood invasive pneumococcal disease in the post-PCV13 era: an international whole genome sequencing study. Lancet Infect Dis 2019 [In revision].

[18] Page AJ, De Silva N, Hunt M, Quail MA, Parkhill J, Harris SR, et al. Robust highthroughput prokaryote de novo assembly and improvement pipeline for Illumina data. Microb Genom 2016;2:e000083.

[19] Page Andrew J, Taylor Ben, Keane Jacqueline A. Multilocus sequence typing by blast from de novo assemblies against PubMLST. J Open Source Softw 2016. https://doi. org/10.21105/joss.00118.

[20] Epping L, van Tonder AJ, Gladstone RA, The Global Pneumococcal Sequencing Consortium, Bentley SD, Page AJ, et al. SeroBA: rapid high-throughput serotyping of Streptococcus pneumoniae from whole genome sequence data. Microb Genom 2018;4. https://doi.org/10.1099/mgen.0.000186.

[21] Li Y, Metcalf BJ, Chochua S, Li Z, Gertz Jr RE, Walker H, et al. Penicillin-binding protein Transpeptidase signatures for tracking and predicting beta-lactam resistance levels in Streptococcus pneumoniae. MBio 2016;7. https://doi.org/10.1128/mBio.00756-16.

[22] Li Y, Metcalf BJ, Chochua S, Li Z, Gertz Jr RE, Walker H, et al. Validation of $\beta$-lactam minimum inhibitory concentration predictions for pneumococcal isolates with newly encountered penicillin binding protein (PBP) sequences. BMC Genomics 2017; 18:621.

[23] Lees JA, Harris SR, Tonkin-Hill G, Gladstone RA, Lo SW, Weiser JN, et al. Fast and flexible bacterial genomic epidemiology with PopPUNK. Genome Res 2019;29:304-16.

[24] Cheng L, Connor TR, Sirén J, Aanensen DM, Corander J. Hierarchical and spatially explicit clustering of DNA sequences with BAPS software. Mol Biol Evol 2013;30: 1224-8.

[25] Mostowy R, Croucher NJ, Andam CP, Corander J, Hanage WP, Marttinen P. Efficient inference of recent and ancestral recombination within bacterial populations. Mol Biol Evol 2017;34:1167-82.

[26] Croucher NJ, Page AJ, Connor TR, Delaney AJ, Keane JA, Bentley SD, et al. Rapid phylogenetic analysis of large samples of recombinant bacterial whole genome sequences using Gubbins. Nucleic Acids Res 2015;43:e15.

[27] Page AJ, Cummins CA, Hunt M, Wong VK, Reuter S, Holden MTG, et al. Roary: rapid large-scale prokaryote pan genome analysis. Bioinformatics 2015;31:3691-3.

[28] Oksanen J, Guillaume Blanchet F, Kindt R, Legendre P, Wagner HH. Vegan: Community ecology package. R package version 2.4-4; 2013 https://CRAN.R-project.org/ package $=$ vegan.

[29] Lees JA, Galardini M, Bentley SD, Weiser JN, Corander J. Pyseer: a comprehensive tool for microbial pangenome-wide association studies. Bioinformatics 2018;34:4310-2.

[30] Weinberger DM, Grant LR, Weatherholtz RC, Warren JL, O'Brien KL, Hammitt LL. Relating pneumococcal carriage among children to disease rates among adults before and after the introduction of conjugate vaccines. Am J Epidemiol 2016;183:1055-62.

[31] Lees J, Ferwerda B, Kremer PHC, Wheeler NE, Serón MV, Croucher NJ, et al. Joint sequencing of human and pathogen genomes reveals the genetics of pneumococcal meningitis. bioRxiv 2018:386078. https://doi.org/10.1101/386078.

[32] McGee L, McDougal L, Zhou J, Spratt BG, Tenover FC, George R, et al. Nomenclature of major antimicrobial-resistant clones of Streptococcus pneumoniae defined by the pneumococcal molecular epidemiology network. J Clin Microbiol 2001;39:2565-71.
[33] Azarian T, Grant LR, Arnold BJ, Hammitt LL, Reid R, Santosham M, et al. The impact of serotype-specific vaccination on phylodynamic parameters of Streptococcus pneumoniae and the pneumococcal pan-genome. PLoS Pathog 2018;14:e1006966.

[34] Lehtinen S, Blanquart F, Croucher NJ, Turner P, Lipsitch M, Fraser C. Evolution of antibiotic resistance is linked to any genetic mechanism affecting bacterial duration of carriage. Proc Natl Acad Sci U S A 2017;114:1075-80.

[35] Croucher NJ, Hanage WP, Harris SR, McGee L, van der Linden M, de Lencastre H, et al Variable recombination dynamics during the emergence, transmission and "disarming” of a multidrug-resistant pneumococcal clone. BMC Biol 2014;12:49.

[36] Azarian T, Martinez PPP, Arnold BJ, Grant LR, Corander J, Fraser C, et al. Prediction of post-vaccine population structure of Streptococcus pneumoniae using accessory gene frequencies. bioRxiv 2018:420315. https://doi.org/10.1101/420315.

[37] Croucher NJ, Finkelstein JA, Pelton SI, Mitchell PK, Lee GM, Parkhill J, et al. Population genomics of post-vaccine changes in pneumococcal epidemiology. Nat Genet 2013; 45:656-63.

[38] Pillai D, Shahinas D, Buzina A, Pollock R, Lau R, Khairnar K, et al. Genome-wide dissection of globally emergent multi-drug resistant serotype 19A Streptococcus pneumoniae. BMC Genomics 2009;10:642.

[39] Sandgren A, Sjostrom K, Olsson-Liljequist B, Christensson B, Samuelsson A, Kronval $\mathrm{G}$, et al. Effect of clonal and serotype-specific properties on the invasive capacity of Streptococcus pneumoniae. J Infect Dis 2004;189:785-96.

[40] Sá-Leão R, Pinto F, Aguiar S, Nunes S, Carriço JA, Frazão N, et al. Analysis of invasiveness of pneumococcal serotypes and clones circulating in Portugal before widespread use of conjugate vaccines reveals heterogeneous behavior of clones expressing the same serotype. J Clin Microbiol 2011;49:1369-75.

[41] Browall S, Norman M, Tångrot J, Galanis I, Sjöström K, Dagerhamn J, et al. Intraclonal variations among Streptococcus pneumoniae isolates influence the likelihood of invasive disease in children. J Infect Dis 2014;209:377-88.

[42] van Tonder AJ, Bray JE, Quirk SJ, Haraldsson G, Jolley KA, Maiden MCJ, et al. Putatively novel serotypes and the potential for reduced vaccine effectiveness: capsular locus diversity revealed among 5405 pneumococcal genomes. Microb Genom 2016; 2:000090.

[43] Park IH, Pritchard DG, Cartee R, Brandao A, Brandileone MCC, Nahm MH. Discovery of a new capsular serotype (6C) within Serogroup 6 of Streptococcus pneumoniae. J Clin Microbiol 2007;45:1225-33.

[44] van Tonder AJ, Gladstone RA, Lo SW, Nahm MH, du Plessis M, Cornick J, et al. Putative novel cps loci in a large global collection of pneumococci; 2019 [Accepted MGen n.d.].

[45] Lees JA, Kremer PHC, Manso AS, Croucher NJ, Ferwerda B, Serón MV, et al. Large scale genomic analysis shows no evidence for pathogen adaptation between the blood and cerebrospinal fluid niches during bacterial meningitis. Microbial Genomics 2017;3:e000103.

[46] Cremers AJH, Mobegi FM, van der Gaast-de Jongh C, van Weert M, van Opzeeland FJ, Vehkala M, et al. The contribution of genetic variation of Streptococcus pneumoniae to the clinical manifestation of invasive pneumococcal disease. Clin Infect Dis 2019;68:9-61. 